\title{
Neurotropic activity and safety of methylene-cycloalkylacetate (MCA) derivative 3-(3-allyl-2-methylenecyclohexyl) propanoic acid
}

Adi Lahiani ${ }^{\ddagger}$, Dikla Hacham-Geula ${ }^{\ddagger}$, David Lankri ${ }^{\dagger}$, Susan Cornell-Kennon ${ }^{+}$, Erik M. Schaefer, ${ }^{+}$ Dmitry Tsvelikhovsky ${ }^{\dagger *}$, and Philip Lazarovici ${ }^{*}$

The Institute for Drug Research, Division of Medicinal Chemistry, School of Pharmacy, Faculty of Medicine, The Hebrew University of Jerusalem, Jerusalem 9112102, Israel.

*The Institute for Drug Research, Division of Pharmacology, School of Pharmacy, Faculty of Medicine, The Hebrew University of Jerusalem, Jerusalem 9112102, Israel.

${ }^{+}$AssayQuant Technologies Inc. 260 Cedar Hill Street, Marlboro, MA 01752, USA

\section{Supporting Information}

\section{Table of contents:}

Sensory motor/neurological performance $\quad$ S1

DiscoverX's SAFETY scan methods and individual target results S3 $\begin{array}{ll}\text { GPCR-induced cAMP modulation } & \text { S3 }\end{array}$ GPCR-induced Calcium Mobilization $\quad$ S4 Nuclear Hormone Receptor Assay $\quad$ S5 KINOMEscan binding assay $\quad$ S6 $\begin{array}{ll}\text { Ion channel assays } & \text { S7 }\end{array}$ $\begin{array}{ll}\text { Transporter assays } & \text { S8 }\end{array}$ Enzymatic assays $\quad$ S8

$\begin{array}{ll}\text { Individual target results (Figures Sup.1 and 2) } & \text { S10-14 }\end{array}$

MCA-13 safety by in vitro evaluation using PhosphoSens ${ }^{\circledR}$ CSoxbased kinase $\begin{array}{ll}\text { and phosphatase assays } & \text { S15 }\end{array}$

Kinase Activity Measurements $\quad$ S15

Progress Curves for Kinases that stimulate neurite outgrowth (Figure S3-2) $\quad$ S17-19 


\section{$\underline{\text { Sensory motor/neurological performance }}$}

The performance was weekly measured using several routine motor tests, but no neurological losses were observed (Table 1). Sensory motor tests were scored individually and according to the neurologic function severity score (NSS). The data from each individual test and the total score calculated from individual tests that make up the mNSS were measured. The following tests were used:

a. Beam balance test -mice were placed on a $2.5 \mathrm{~cm}$ wide beam for 60 seconds. A normal response is balance with steady posture for 60 seconds (a score of 0 ). Deficits are scored if the rat: 1. Grasps the side of the beam (score of 1); 2. Hugs the beam and one limb falls down from the beam (score of 2); 3. Hugs the beam and two limbs fall off the beam (score of 3); 4. Try to balance on the beam, but falls off from 60 seconds (score of 4); 5 . Try to balance on the beam but falls off from 60 seconds (score of 5); 6. Falls off with no attempt to balance or hang on the beam in less than 20 seconds (score of 6 ).

b. Foot fault test- dysfunction of forelimb movement, while walking on elevated metal grids. The animal was placed on horizontal grids $(85 \times 26 \times 20) \mathrm{cm}$ with a glass enclosure for observation. With each weight bearing step, the forelimb can fall or slip between the metal support bars, an event recorded as a foot fault. The total number of forelimb steps and the total number of foot faults were counted. The percentage of forelimb foot faults to the total steps that occurred within 2 minutes represent the test results.

c. Hind limb placing test- This assay monitors reposition of hind limbs placed down and away from the table edge. The ability to retrieve and place the hind limbs back onto the table was measured. Immediate and complete limb retrieval was scored 0 , delayed ( $>2$ seconds) limb retrieval and/or interspersed flailing was scored 1, and no limb retrieval was scored 2;

d. Increasing platform angle to slide test- This assay measures the strength and stability of resistance to slide down an inclined platform. It deploys a clean wooden board $(60 \mathrm{X} 40 \mathrm{~cm})$ positioned horizontally. Mice were placed on the board with head facing up the increasing incline. The board then swings to increase the degree of inclination the rat experiences until slipping down the board feet first. The degree of the angle at which the rat relinquishes their grip/stance on the board (slips down) represents the test results; 
e. Forelimb whole body suspension test- This assay measures grip strength by duration of suspension by forepaws. The rat is suspended on a metal bar (diameter of $5 \mathrm{~mm}$ ) which it tightly holds onto by its forelimbs. The time (seconds) during which each rat could sustain its body weight while holding onto the bar is measured. The mice drop from the bar onto soft material with no harm. The test was repeated three times and a mean result per rat was used as the score.

Cumulatively these sensory motor/neurological findings indicate acute tolerability of $250 \mathrm{mg} / \mathrm{kg}$ $\underline{H U-M C A-13 \text { iv injection in Male C57BL/6 mice }}$

\begin{tabular}{|c|c|c|c|c|c|c|c|}
\hline \multirow[b]{2}{*}{ NSS } & \multicolumn{3}{|c|}{ DM SO } & \multicolumn{3}{|c|}{ HU-MCA-13 } & \multirow[b]{3}{*}{0} \\
\hline & & & & & & & \\
\hline Day 0 & 0 & 0 & 0 & 0 & 0 & 0 & \\
\hline Day 1 & 0 & 0 & 0 & 0 & 0 & 0 & 0 \\
\hline Day 5 & 0 & 0 & 0 & 0 & 0 & 0 & 0 \\
\hline \multicolumn{8}{|c|}{ Foot fault (\%) } \\
\hline Day 0 & $0 / 24$ & $0 / 33$ & $0 / 30$ & $0 / 34$ & $0 / 41$ & $0 / 38$ & $1 / 48$ \\
\hline Day 1 & $0 / 52$ & $0 / 59$ & $0 / 67$ & $0 / 20$ & $0 / 15$ & $0 / 34$ & $0 / 14$ \\
\hline Day 5 & $0 / 48$ & $0 / 54$ & $0 / 33$ & $2 / 50$ & $1 / 47$ & $0 / 48$ & $0 / 36$ \\
\hline \multicolumn{8}{|c|}{$H$ ind limb } \\
\hline Day 0 & 0 & 0 & 0 & 0 & 0 & 0 & 0 \\
\hline Day 1 & 0 & 0 & 0 & 0 & 0 & 0 & 0 \\
\hline Day 5 & 0 & 0 & 0 & 0 & 0 & 0 & 0 \\
\hline \multicolumn{8}{|c|}{ Beam balance } \\
\hline Day 0 & 0 & 0 & 0 & 0 & 0 & 0 & 0 \\
\hline Day 1 & 0 & 0 & 0 & 0 & 0 & 0 & 0 \\
\hline Day 5 & 0 & 0 & 0 & 0 & 0 & 0 & 0 \\
\hline \multicolumn{8}{|c|}{ Self-s us pens ion time (seconds) } \\
\hline Day 0 & 10 & 7 & 8 & 8 & 10 & 9 & 10 \\
\hline Day 1 & 8 & 14 & 10 & 14 & 12 & 15 & 12 \\
\hline Day 5 & 9 & 14 & 13 & 13 & 13 & 15 & 14 \\
\hline \multicolumn{8}{|c|}{ Angle of increase (degrees) } \\
\hline Day 0 & 35 & 35 & 35 & 35 & 35 & 35 & 35 \\
\hline Day 1 & 35 & 35 & 35 & 35 & 35 & 35 & 35 \\
\hline Day 5 & 35 & 35 & 35 & 35 & 35 & 35 & 35 \\
\hline
\end{tabular}




\section{DiscoverX's SAFETY scan methods and individual target results}

\section{GPCR-induced cAMP modulation}

cAMP Hunter cell lines were expanded from freezer stocks according to standard procedures. The cells were seeded in a total volume of $20 \mu 1$ into 384 -well microplates and incubated at $37^{\circ} \mathrm{C}$ for the appropriate time, prior to testing. cAMP modulation was determined using the DiscoverX HitHunter cAMP XS+ assay (https://www.discoverx.com/products-applications/camp-assays). For Gs agonist determination, cells were incubated with sample to induce the response. For Gi agonist determination, cells were incubated with sample in the presence of $\mathrm{EC}_{80}$ forskolin to induce the response. Media was aspirated from cells and replaced with $15 \mu 1$ 2:1 HBSS/10 mM Hepes : cAMP XS $+\mathrm{Ab}$ reagent. Intermediate dilution of sample stocks was performed to generate $4 \mathrm{X}$ sample in assay buffer. $4.5 \mu 1$ of $4 \mathrm{X}$ sample was added to cells and incubated at $37{ }^{\circ} \mathrm{C}$ or room temperature for 30 or 60 minutes. Final assay vehicle concentration was 1\%. For antagonist determination, cells were pre-incubated with sample followed by agonist challenge at the $\mathrm{EC}_{80}$ concentration. Media was aspirated from cells and replaced with $10 \mu 1$ 1:1 HBSS/Hepes: cAMP $\mathrm{XS}+\mathrm{Ab}$ reagent. $3.5 \mu \mathrm{l}$ of $4 \mathrm{X}$ compound was added to the cells and incubated at $37^{\circ} \mathrm{C}$ or room

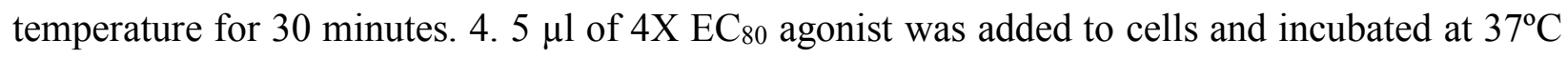
or room temperature for 30 or 60 minutes. For Gi coupled GPCRs, $\mathrm{EC}_{80}$ forskolin was included. After appropriate compound incubation, assay signal was generated through incubation with $20 \mu 1$ cAMP XS+ED/CL lysis cocktail for one hour followed by incubation with $20 \mu 1$ cAMP XS+EA reagent for three hours at room temperature. Microplates were read following signal generation with a PerkinElmer Envision ${ }^{\mathrm{TM}}$ instrument for chemiluminescent signal detection. Compound activity was analyzed using CBIS data analysis suite (ChemInnovation, CA).

For Gs agonist mode assays, percentage activity was calculated using the following formula: $\%$ Activity $=100 \% \times$ (mean RLU of test sample - mean RLU of vehicle control) / (mean RLU of MAX control - mean RLU of vehicle control).

For Gs antagonist mode assays, percentage inhibition was calculated using the following formula: $\%$ Inhibition $=100 \% \times$ ( 1 - (mean RLU of test sample - mean RLU of vehicle control) / (mean RLU of $\mathrm{EC}_{80}$ control - mean RLU of vehicle control).

For Gi agonist mode assays, percentage activity was calculated using the following formula: 
$\%$ Activity $=100 \% \times(1-($ mean RLU of test sample - mean RLU of MAX control) / (mean RLU of vehicle control - mean RLU of MAX control).

For Gi antagonist or negative allosteric mode assays, percentage inhibition was calculated using the following formula:

$\%$ Inhibition $=100 \% \times$ (mean RLU of test sample - mean RLU of $\mathrm{EC}_{80}$ control) $/$ (mean RLU of forskolin positive control - mean RLU of $\mathrm{EC}_{80}$ control).

For primary screens, percent response was capped at $0 \%$ or $100 \%$ where calculated percent response returned a negative value or a value greater than 100, respectively.

\section{GPCR-induced Calcium Mobilization}

Cell lines were expanded from freezer stocks according to standard procedures. Cells $(10,000$ cells/well) were seeded in a total volume of $50 \mu \mathrm{l}$ (200 cells/ $\mu \mathrm{l})$ into black-walled, clear-bottom, Poly-D-lysine coated 384-well microplates and incubated at $37{ }^{\circ} \mathrm{C}$ for the appropriate time prior to testing. DMSO concentration for all readouts was $0.2 \%$. The assays were performed in $1 \mathrm{X}$ Dye Loading Buffer consisting of 1X Dye (DiscoverX, Calcium No WashPLUS kit, Catalog No.900091), 1X Additive A and $2.5 \mathrm{mM}$ fresh Probenecid in HBSS / $20 \mathrm{mM}$ Hepes. The cells were loaded with dye prior to testing. Media was aspirated from cells and replaced with $25 \mu 1$ Dye Loading Buffer. Thereafter, the cells were incubated for 45 minutes at $37^{\circ} \mathrm{C}$ followed by 20 minutes at room temperature. For agonist determination, cells were incubated with sample to induce the response. After dye loading, the cells were removed from the incubator and $25 \mu 1$ of $2 \mathrm{X}$ compound in HBSS / 20 mM Hepes was added using a FLIPR Tetra (MDS). Compound agonist activity was measured on a FLIPR Tetra. Calcium mobilization was monitored for 2 minutes with a 5 second baseline read. For antagonist determination, cells were pre-incubated with sample followed by agonist challenge at the $\mathrm{EC}_{80}$ concentration. After dye loading, the cells were removed from the incubator and $25 \mu 12 \mathrm{X}$ sample was added. Cells were incubated for 30 minutes at room temperature in the dark to equilibrate plate temperature. After incubation, antagonist determination was initiated with addition of $25 \mu \mathrm{l} 1 \mathrm{X}$ compound with $3 \mathrm{X} \mathrm{EC}_{80}$ agonist using FLIPR. Compound antagonist activity was measured on a FLIPR Tetra (MDS). Calcium mobilization was monitored for 2 minutes with a 5 second baseline read. FLIPR read - area under the curve was calculated for the entire two minute read. Compound activity was analyzed using CBIS data analysis suite (ChemInnovation, CA). 
For agonist mode assays, percentage activity was calculated using the following formula:

$\%$ Activity $=100 \% \mathrm{x}$ (mean RFU of test sample - mean RFU of vehicle control) / (mean MAX RFU control ligand - mean RFU of vehicle control).

For antagonist mode assays, percentage inhibition was calculated using the following formula: $\%$ Inhibition $=100 \% \times(1-($ mean RFU of test sample - mean RFU of vehicle control) $/$ (mean RFU of $\mathrm{EC}_{80}$ control - mean RFU of vehicle control).

For Primary screens, percent response was capped at $0 \%$ or $100 \%$ where calculated percent response returned a negative value or a value greater than 100, respectively.

\section{Nuclear Hormone Receptor Assay}

For this assay, (https://www.discoverx.com/products-applications/assay-ready-kits/pathhunternhr-assay-ready-express-kits) DiscoverX PathHunter NHR cell lines were expanded from freezer stocks according to standard procedures. The cells were seeded in a total volume of $20 \mu 1$ into white walled, 384-well microplates and incubated at $37^{\circ} \mathrm{C}$ for the appropriate time prior to testing. Assay media contained charcoal-dextran filtered serum to reduce the level of hormones present. For agonist determination, cells were incubated with sample to induce the response. Intermediate dilution of sample stocks was performed to generate $5 \mathrm{X}$ sample in assay buffer. $3.5 \mu 1$ of $5 \mathrm{X}$ sample was added to cells and incubated at $37^{\circ} \mathrm{C}$ or room temperature for 3-16 hours. Final assay vehicle concentration was 1\%. For antagonist determination, cells were pre-incubated with antagonist followed by agonist challenge at the $\mathrm{EC}_{80}$ concentration. Intermediate dilution of sample stocks was performed to generate $5 \mathrm{X}$ sample in assay buffer. $3.5 \mu 1$ of $5 \mathrm{X}$ sample was added to cells and incubated at $37^{\circ} \mathrm{C}$ or room temperature for 60 minutes. Vehicle concentration was $1 \%$. 4. $5 \mu \mathrm{l}$ of $6 \mathrm{X} \mathrm{EC}_{80}$ agonist in assay buffer was added to the cells and incubated at $37^{\circ} \mathrm{C}$ or room temperature for 3-16 hours. Assay signal was generated through a single addition of 12.5 or $15 \mu \mathrm{l}(50 \% \mathrm{v} / \mathrm{v})$ of PathHunter Detection reagent cocktail, followed by a one-hour incubation at room temperature. Microplates were read following signal generation with a PerkinElmer Envision $^{\mathrm{TM}}$ instrument for chemiluminescent signal detection. Compound activity was analyzed using CBIS data analysis suite (ChemInnovation, CA).

For agonist mode assays, percentage activity was calculated using the following formula: $\%$ Activity $=100 \% \mathrm{x}$ (mean RLU of test sample - mean RLU of vehicle control) / (mean MAX control ligand - mean RLU of vehicle control). 
For antagonist mode assays, percentage inhibition was calculated using the following formula: $\%$ Inhibition $=100 \% \times(1-($ mean RLU of test sample - mean RLU of vehicle control $) /($ mean RLU of $\mathrm{EC}_{80}$ control - mean RLU of vehicle control).

Note that for select assays, the ligand response produces an decrease in receptor activity (inverse agonist with a constitutively active target). For those assays inverse agonist activity was calculated using the following formula:

$\%$ Inverse Agonist Activity $=100 \%$ x (mean RLU of vehicle control - mean RLU of test sample) / (mean RLU of vehicle control- mean RLU of MAX control).

For Primary screens, percent response was capped at $0 \%$ or $100 \%$ where calculated percent response returned a negative value or a value greater than 100 , respectively.

\section{KINOMEscan binding assay}

Kinase-tagged T7 phage strains were grown in parallel in 24-well blocks in an E. coli host derived from the BL21 strain. E. coli were grown to log-phase and infected with T7 phage from a frozen stock (multiplicity of infection $=0.4)$ and incubated with shaking at $32^{\circ} \mathrm{C}$ until lysis $(90-150$ minutes). The lysates were centrifuged $(6,000 \mathrm{xg})$ and filtered $(0.2 \mu \mathrm{m})$ to remove cell debris. The kinases were produced in HEK-293 cells and subsequently tagged with DNA for qPCR detection. Capture Ligand Production Streptavidin-coated magnetic beads were treated with biotinylated small molecule ligands for 30 minutes at room temperature to generate affinity resins for kinase assays. The liganded beads were blocked with excess biotin and washed with blocking buffer [SeaBlock (Pierce- Thermo Fisher Scientific, Waltham, MA, USA), 1\% BSA, 0.05\% Tween 20, 1 $\mathrm{mM}$ DTT] to remove unbound ligand and to reduce non-specific phage binding. Binding reactions were assembled by combining kinases, ligand- affinity beads, and test compounds in $1 \mathrm{X}$ binding buffer (20\% SeaBlock, 0.17X PBS, 0.05\% Tween 20, 6 mM DTT). All reactions were performed in polypropylene 384-well plates in a final volume of $0.02 \mathrm{ml}$. The assay plates were incubated at room temperature with shaking for 1 hour and the affinity beads were washed with wash buffer (1x PBS, 0.05\% Tween 20). The beads were then re-suspended in elution buffer (1x PBS, 0.05\% Tween 20, $0.5 \mu \mathrm{M}$ non-biotinylated affinity ligand) and incubated at room temperature with shaking for 30 minutes. The kinase concentration in the eluates was measured by qPCR. The kinase concentration in the eluates was measured by qPCR. qPCR reactions were assembled by adding $2.5 \mu \mathrm{L}$ of kinase eluate to $7.5 \mu \mathrm{l}$ of qPCR master mix containing $0.15 \mu \mathrm{M}$ amplicon primers and 
$0.15 \mu \mathrm{M}$ amplicon probe. The qPCR protocol consisted of a 10 -minute hot start at $95{ }^{\circ} \mathrm{C}$, followed by 35 cycles of $95{ }^{\circ} \mathrm{C}$ for 15 seconds, $60{ }^{\circ} \mathrm{C}$ for 1 minute. Data Analysis: (Percent Response Calculation equal (HU-MCA13 signal - positive control signal/ DMSO signal - positive control signal) $\times 100$; Percent of Control was converted to Percent Response using the formula: Percent Response $=(100-$ Percent Control). For Primary screens, percent response was capped at $0 \%$ or $100 \%$ where calculated percent response returned a negative value or a value greater than 100 , respectively. Binding constants (Kds) were calculated with a standard dose-response curve using the Hill equation: Response $=$ Background + Signal - Background $1+($ Kd Hill Slope/Dose Hill Slope). The Hill Slope was set to -1 . Curves were fitted using a non-linear least square fit with the Levenberg-Marquardt algorithm.

\section{Ion channel assays}

Cell lines were expanded from freezer stocks according to standard procedures. The cells were plated in a total volume of $20 \mu \mathrm{L}$ into black-walled, clear-bottom, Poly-D-lysine coated 384-well microplates and incubated at $37{ }^{\circ} \mathrm{C}$ for the appropriate time prior to testing. The assay was performed in 1X Dye Loading Buffer consisting of 1X Dye, and $2.5 \mathrm{mM}$ freshly prepared Probenecid when applicable. The cells were incubated for 30-60 minutes at $37{ }^{\circ} \mathrm{C}$. For agonist determination, the cells were incubated with sample to induce response. Intermediate dilution of sample stocks was performed to generate 2 - 5X sample in assay buffer. $10-25 \mu \mathrm{L}$ of 2 - 5X sample was added to cells and incubated at $37^{\circ} \mathrm{C}$ or room temperature for 30 minutes. Final assay vehicle concentration was $1 \%$. For antagonist determination, cells were pre-incubated with the sample. Intermediate dilution of sample stocks was performed to generate 2 - $5 \mathrm{X}$ sample in assay buffer. After dye loading, the cells were removed from the incubator and 10 - $25 \mu \mathrm{L} 2$ - 5X sample was added to cells in the presence of $\mathrm{EC}_{80}$ agonist when appropriate. Cells were incubated for 30 minutes at room temperature in the dark to equilibrate plate temperature. Vehicle concentration was $1 \%$. Compound activity was measured on a FLIPR Tetra (MDS). Compound activity was analyzed using CBIS data analysis suite (ChemInnovation, CA).

For agonist mode assays, percentage activity was calculated using the following formula: Activity $(\%)=100 \% \times$ (mean RLU of test sample - mean RLU of vehicle control) / (mean MAX control ligand - mean RLU of vehicle control). 
For antagonist percentage inhibition was calculated using the following formula: Inhibition (\%) $=100 \% \times$ x 11 - (mean RLU of test sample - mean RLU of vehicle control) / (mean RLU of EC 80 control - mean RLU of vehicle control)]. For Primary screens, percent response was capped at $0 \%$ or $100 \%$ where calculated percent response returned a negative value or a value greater than 100 , respectively.

\section{Transporter assays}

The cell lines were expanded from freezer stocks according to standard procedures. Cells were plated in a total volume of $25 \mu \mathrm{L}$ into black-walled, clear-bottom, Poly-D-lysine coated 384-well microplates and incubated at $37^{\circ} \mathrm{C}$ for the appropriate time prior to testing. After cell plating and incubation, media was removed and $25 \mu \mathrm{L}$ of $1 \mathrm{X}$ compound in $1 \mathrm{X} \mathrm{HBSS} / 0.1 \%$ BAS was added. Compounds were incubated with cells at $37^{\circ} \mathrm{C}$ for 30 minutes. Assays were performed in $1 \mathrm{X}$ Dye Loading Buffer consisting of 1X Dye, 1X HBSS / 20 mM Hepes. After compound incubation, 25 $\mu \mathrm{L}$ of $1 \mathrm{X}$ dye was added to wells. Thereafter the cells were incubated for $30-60$ minutes at $37^{\circ} \mathrm{C}$. After dye incubation, microplates were transferred to a PerkinElmer Envision ${ }^{\mathrm{TM}}$ instrument for fluorescence signal detection. Compound activity was analyzed using CBIS data analysis suite (ChemInnovation, CA, USA). For blocker mode assays, percentage inhibition was calculated using the following formula: Inhibition $(\%)=100 \%$ x $[1$ - (mean RLU of test sample - mean RLU of vehicle control) / (mean RLU of positive control - mean RLU of vehicle control)]. For Primary screens, percent response was capped at $0 \%$ or $100 \%$ where calculated percent response returned a negative value or a value greater than 100 , respectively.

\section{Enzymatic assays}

Enzyme preparations were purchased from different companies: AChE (R\&D Systems, Minneapolis, MN, USA), COX1 and COX2 (BPS Bioscience, San Diego, CA, USA), MAO-A (Sigma-Aldrich-Merck), PDE3A and PDE4D2 (Signal Chem, Richmond, BC, Canada). The enzymatic assays determined the enzymatic activity by measuring either the consumption of substrate or production of product over time. Different detection methods were used in each enzymatic assay to measure the concentrations of substrates and products: 1. AChE: enzyme and test compound were preincubated for 15 minutes at room temperature before substrate addition. Acetylthiocholine and DTNB were added and incubated at room temperature for 30 minutes. 
Signal was detected by measuring absorbance at $405 \mathrm{~nm}$. 2. COX1 \& COX2: Enzyme stocks were diluted in the assay buffer $(40 \mathrm{mM}$ Tris-HCl, $1 \mathrm{X}$ PBS, $0.5 \mathrm{mM}$ Phenol, 0.01\% Tween-20 + 100 $\mathrm{nM}$ Hematin) and allowed to equilibrate with compounds at room temperature for 30 minutes (binding incubation). Arachidonic acid $(1.7 \mu \mathrm{M})$ and Ampliflu Red $(2.5 \mu \mathrm{M})$ were prepared and dispensed into a reaction plate. Plates were read immediately on a fluorimeter with the emission detection at $590 \mathrm{~nm}$ and excitation wavelength $544 \mathrm{~nm}$. 3. MAO-A: Enzyme and test compound were preincubated for 15 minutes at $37^{\circ} \mathrm{C}$ before substrate addition. The reaction was initiated by addition of kynuramine and incubated at $37^{\circ} \mathrm{C}$ for 30 minutes. The reaction was terminated by addition of $\mathrm{NaOH}$. The amount of 4-hydroquioline formed was determined through spectrofluorimetric readout with the emission detection at $380 \mathrm{~nm}$ and excitation wavelength 310 nm. 4. PDE3A \& PDE4D2: Enzyme and test compound were preincubated for 15 minutes at room temperature before substrate addition. cAMP substrate (at a concentration equal to $\mathrm{EC}_{80}$ ) was added and incubated at room temperature for 30 minutes. Enzyme reaction was terminated by addition of 9 mM IBMX. Signal was detected using the HitHunter®cAMP detection kit. For each assay, microplates were transferred to a PerkinElmer Envision ${ }^{\mathrm{TM}}$ instrument and readout as described. Compound activity was analyzed using CBIS data analysis suite (ChemInnovation, CA). For enzyme activity assays, percentage inhibition was calculated using the following formula: Inhibition (\%) $=100 \%$ x [1 - (mean RLU of test sample - mean RLU of vehicle control) / (mean RLU of positive control - mean RLU of vehicle control)]. For Primary screens, percent response was capped at $0 \%$ or $100 \%$ where calculated percent response returned a negative value or a value greater than 100 , respectively. 
Figure Sup. 1 - Individual target results - Screening of HU-MCA-13 dose-response in vitro, on selected GPCRs, ion channels, kinases, nuclear hormone receptors, enzymes and neurotransmitter transporters.

Data (mean of triplicates) shown was normalized to the maximal and minimal response observed in the presence of control reference ligand and DMSO vehicle respectively (y-axis) and is plotted against the corresponding HU-MCA-13 compound concentration (nM) in log scale (x-axis).

3-(3-allyl-2-methylenecyclohexyl)propanoic acid ADORA2A Agonis
RC50 (UM) $>10$

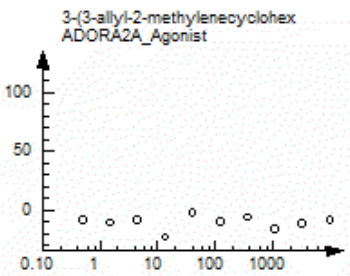

3-(3-allyl-2-methylenecyclohexyl)propanoic acid ADRA2A Agonist
RCSO (UM) $>10$

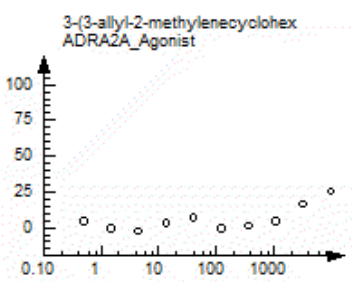

3-(3-allyl-2-methylenecyclohexyl)propanoic acid ADRB2 Agonist
RCSO (UM) $>10$

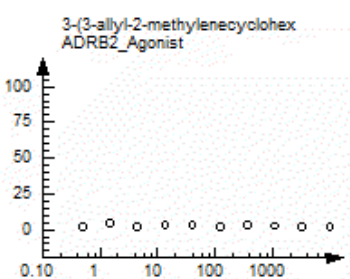

3-(3-allyl-2-methylenecyclohexyl)propanoic acid CCKAR Agonist

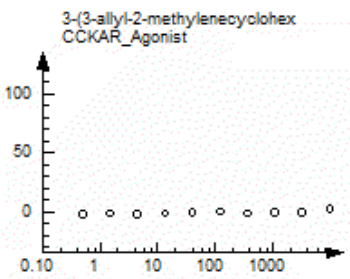

3-(3-allyl-2-methylenecyclohexyl)propanoic acid ADORA2A Antagonist

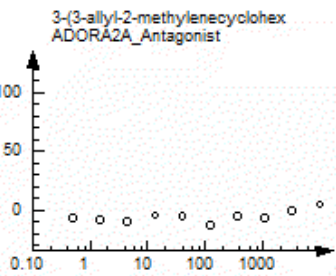

3-(3-ally-2-methylenecyclohexyl)propanoic acid ADRA2A Antagonist
RC50 (UM) $>10$

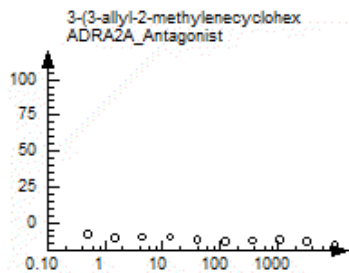

3-(3-allyl-2-methylenecyclohexyl)propanoic acid ADRB2 Antagonist
RC50 (UM) $>10$

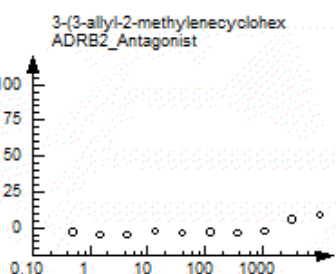

3-(3-allyl-2-methylenecyclohexyl)propanoic acid CCKAR Antagonis
RC50 (UM) $>10$

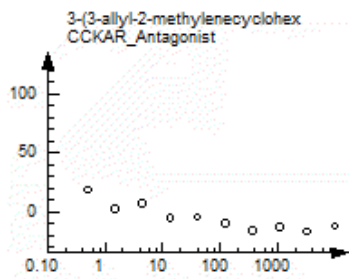

3-(3-allyl-2-methylenecyclohexyl)propanoic acid RC50 (UM) $>10$
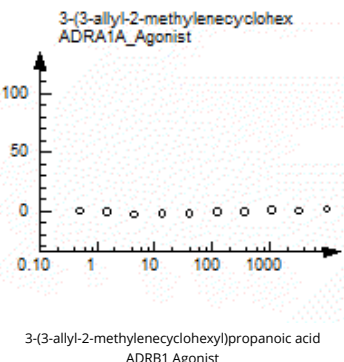
ADRB1 Agonist
RC50 (UM) $>10$
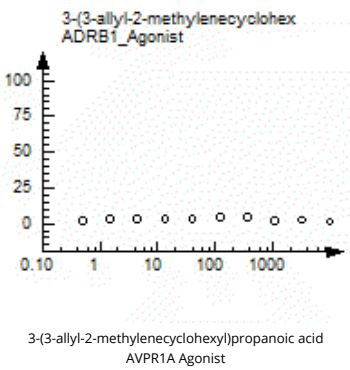
$R C 50(M M)>10$

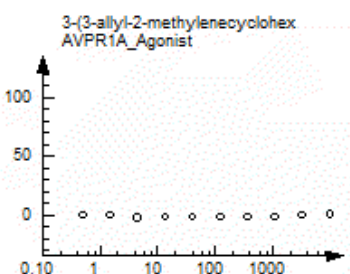

3-(3-allyl-2-methylenecyclohexyl)propanoic acid CHRM1 Agonis

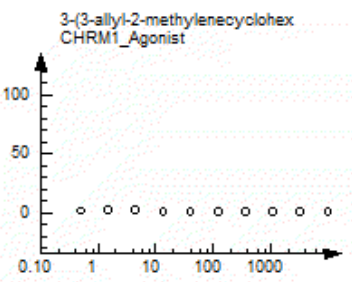

3-(3-allyl-2-methylenecyclohexyl)propanoic acid ADRA1A Antagonis
RC50 (UM) $>10$

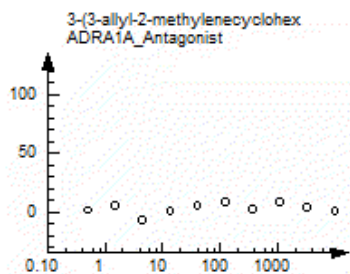

3-(3-allyl-2-methylenecyclohexyl)propanoic acid
ADRB1 Antagonist $\mathrm{RC} 50(\mathrm{UM})>10$

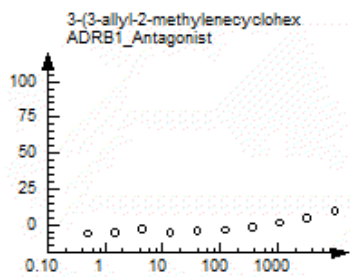

3-(3-ally-2-methylenecyclohexy))propanoic acid AVPR1A Antagonist
RC50 (UM) $>10$

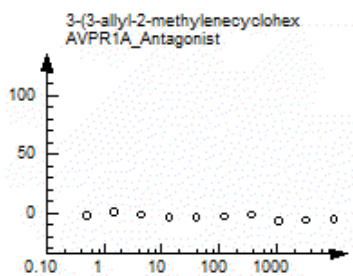

3-(3-allyl-2-methylenecyclohexyl)propanoic acid CHRM1 Antagonis

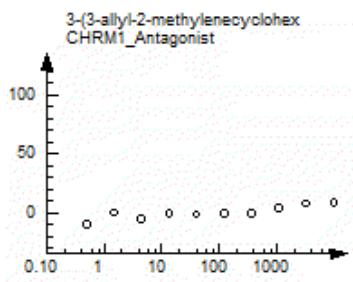


Figure Sup. 1 (cont.)

3-(3-allyl-2-methylenecyclohexyl)propanoic acid
CHRM2 Agonist CHRM2 Agonist
$\mathrm{RC50}(\mathrm{UM})>10$

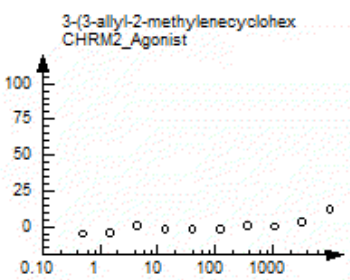

3-(3-allyl-2-methylenecyclohexyl)propanoic acid CNR1 Agonist
RC50 (uM) $>10$

3-(3-allyl-2-methylenecyclohex

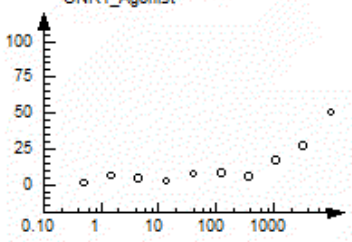

3-(3-ally|-2-methylenecyclohexyl)propanoic acid DRD1 Agonist
RC50 (UM) $>10$

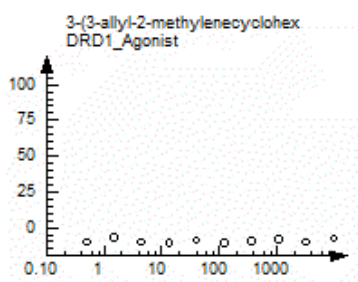

3-(3-allyl-2-methylenecyclohexyl)propanoic acid EDNRA Agonist
RC50 (UM) $>10$

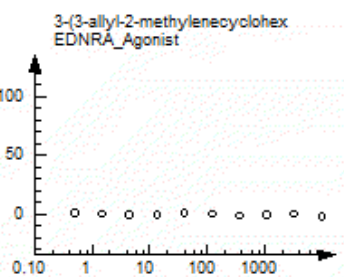

3-(3-allyl-2-methylenecyclohexyl)propanoic acid CHRM2 Antagonist
RC50 (UM) $>10$

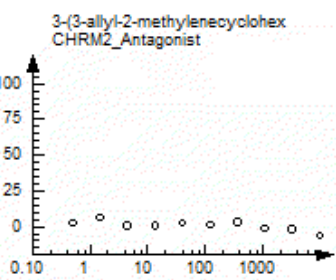

3-(3-allyl-2-methylenecyclohexyl)propanoic acid CNR1 Antagonist
RC50 (uM $>10$

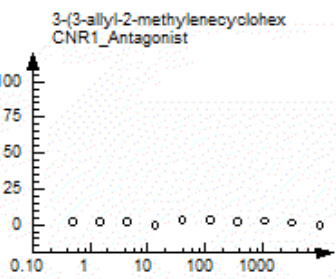

3-(3-allyl-2-methylenecyclohexyl)propanoic acid DRD1 Antagonist
RC50 (UM) $>10$

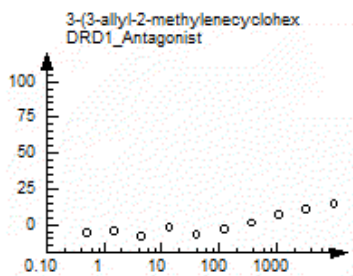

3-(3-ally-2-methylenecyclohexyl)propanoic acid EDNRA Antagonist
RC50 $(\mathrm{UM})>10$

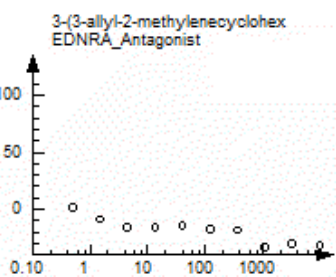

3-(3-allyl-2-methylenecyclohexyl)propanoic acid CHRM3 Agonist

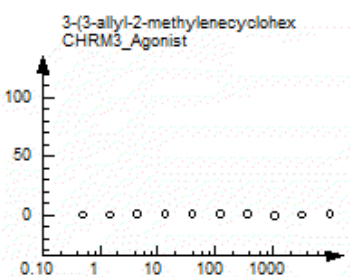

3-(3-allyl-2-methylenecyclohexy)|)propanoic acid CNR2 Agonist

3-(3-allyl-2-methylenecyclohex
CNR2 Agonist

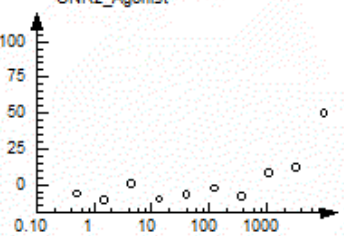

3-(3-allyl-2-methylenecyclohexyl)propanoic acid RC50 (UM) $>10$

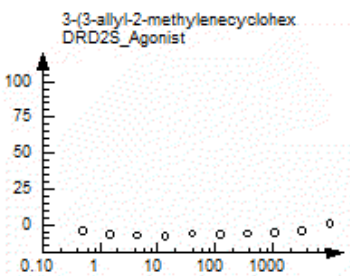

3-(3-allyl-2-methylenecyclohexyl)propanoic acid HRH1 Agonist
RC50 (MM) $>10$

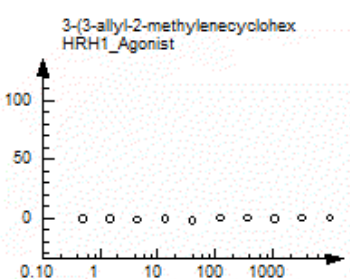

3-(3-ally-2-methylenecyclohexyl)propanoic acid CHRM3 Antagonist
RC50 (UM) $>10$

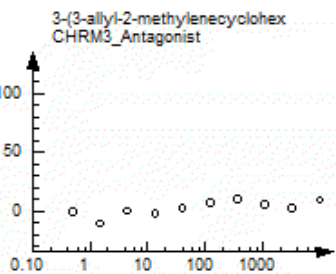

3-3-allyl-2-methylenecyclohexyl)propanoic acid CNR2 Antagonist
RC50 (uM)> 10

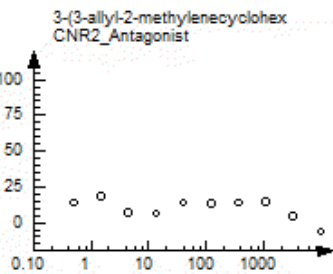

3-(3-allyl-2-methylenecyclohexyl)propanoic acid $\mathrm{RC} 50(\mathrm{UM})>10$

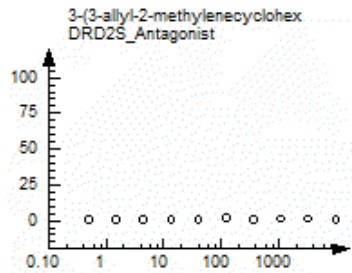

3-(3-allyl-2-methylenecyclohexyl)propanoic acid
HRH1 Antagonist RC50 (uM) $>10$

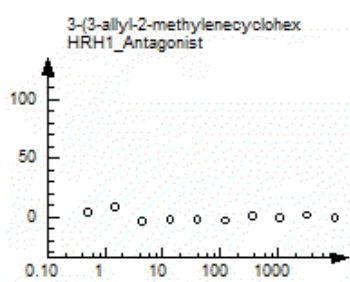


Figure Sup. 1 (cont.)
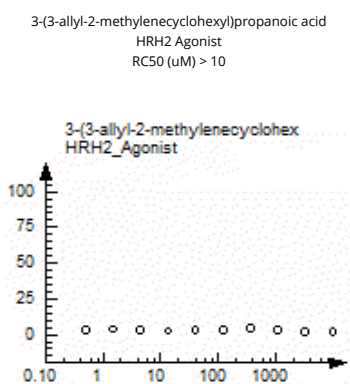

3-(3-allyl-2-methylenecyclohexyl)propanoic acid HTR1B Agonis

3-(3-ally|-2-methylenecyclohex

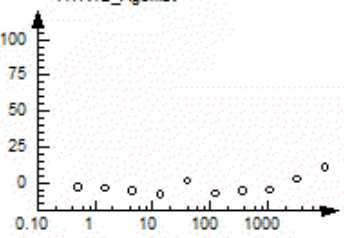

3-(3-ally-2-methylenecyclohexyl)propanoic acid HTR2B Agonist
RC50 (UM) $>10$

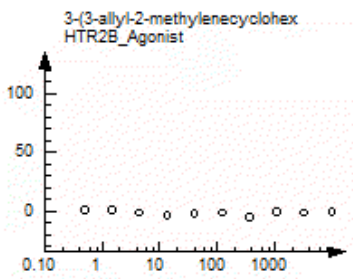

3-(3-allyl-2-methylenecyclohexyl)propanoic acid
OPRK1 Agonist OPRKI Agonist

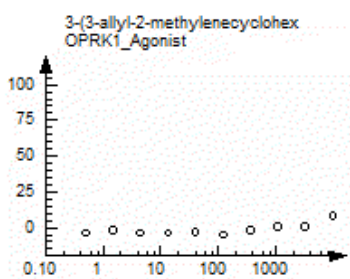

3-(3-allyl-2-methylenecyclohexyl)propanoic acid $\mathrm{HRH} 2$ Antagonist
$\mathrm{RC} 50(\mathrm{UM})>10$

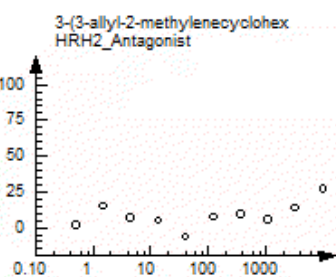

3-(3-allyl-2-methylenecyclohexyl)propanoic acid HTR1B Antagonist
RCSO (uM) $>10$

3-(3-allyl-2-methylenecyclohex
HTR1B

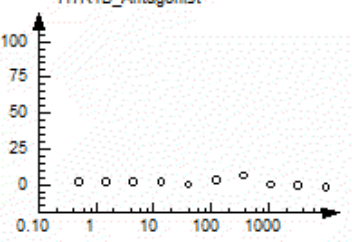

3-(3-allyl-2-methylenecyclohexyl)propanoic acid HTR2B Antagonist
RC50 (uM $)>10$

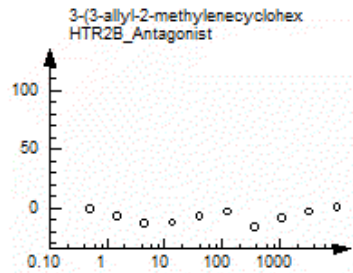

3-(3-allyl-2-methylenecyclohexyl)propanoic acid
OPRK1 Antagonist RCI Antagonist

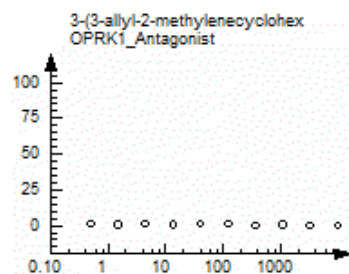

3-(3-allyl-2-methylenecyclohexyl)propanoic acid HTR1A Agonist

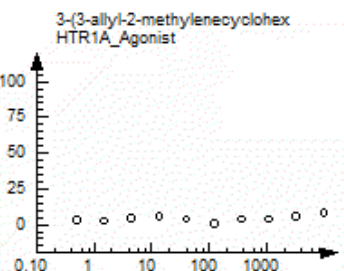

3-(3-allyl-2-methylenecyclohexyl)propanoic acid HTR2A Agonist
RC50 (uM) $>10$

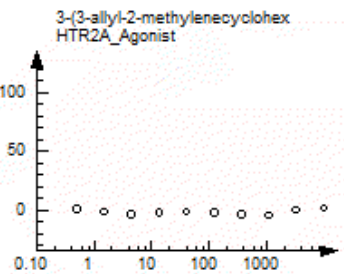

3-(3-allyl-2-methylenecyclohexyl)propanoic acid OPRD1 Agonist
RC50 (uM) $>1$

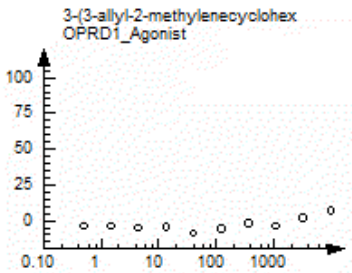

3-(3-ally-2-methylenecyclohexyl)propanoic acid
OPRM1 Agonist PrRT Agonist

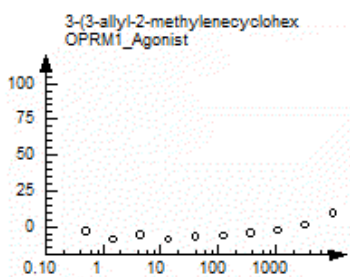

3-(3-ally|-2-methylenecyclohexyl)propanoic acid HTR1A Antagonis
RCSO (uM) $>10$

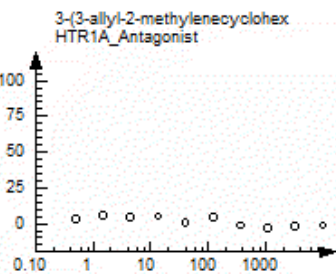

3-(3-allyl-2-methylenecyclohexyl)propanoic acid HTR2A Antagonist

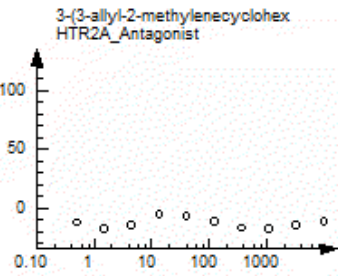

3-(3-allyl-2-methylenecyclohexyl)propanoic acid RC50 (uM) $>10$
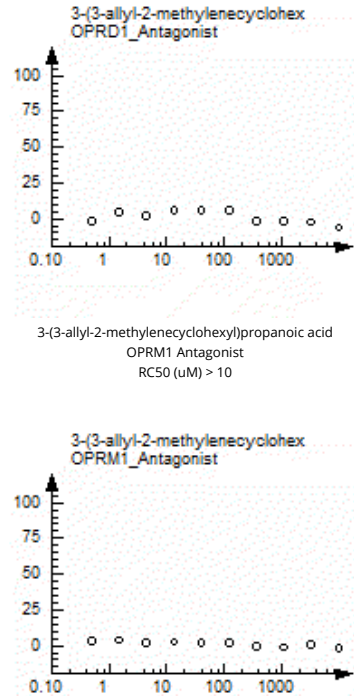
Figure Sup. 1 (cont.)

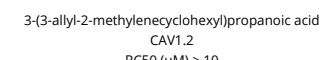
CAV1.2
$\operatorname{RC} 50($ (uM $)>10$

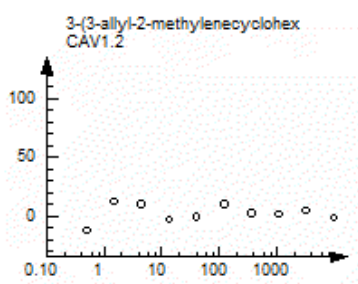

3-(3-allyl-2-methylenecyclohexyl)propanoic acid RTRO (UM) $>10$
RC

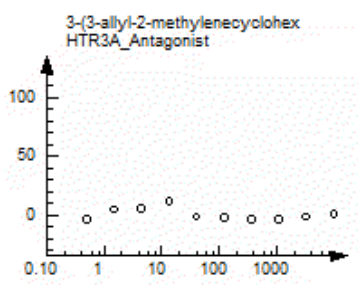

3-(3-ally-2-methylenecyclohexyl)propanoic acid nAChR(a $4 / \mathrm{b} 2)$ Antagonist
RC50 (uM) $>10$
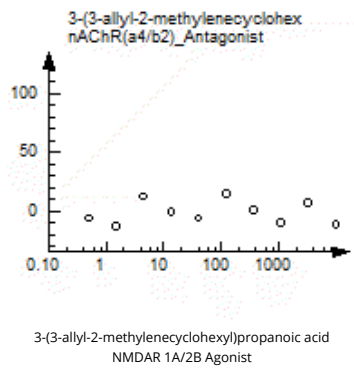
NMDAR 1A/2B Agonist
RC50 (UM) $>10$

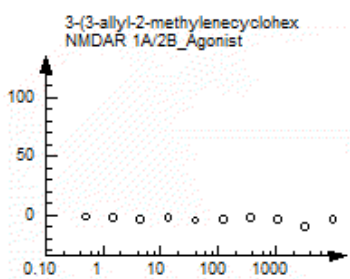

3-(3-allyl-2-methylenecyclohexyl)propanoic acid GABAA Antagonist
RC50 (UM) $>10$

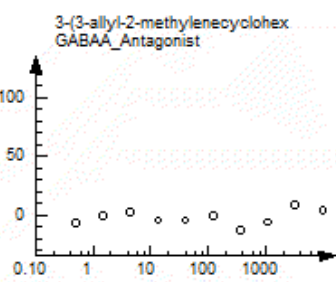

3-(3-ally-2-methylenecyclohexyl)propanoic acid HTR3A Agonist
RC50 (uM) $>10$

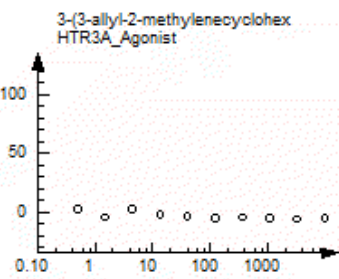

3-(3-allyl-2-methylenecyclohexyl)propanoic acid nAChR(a4/b2) Agonist
RC50 (UM) $>10$
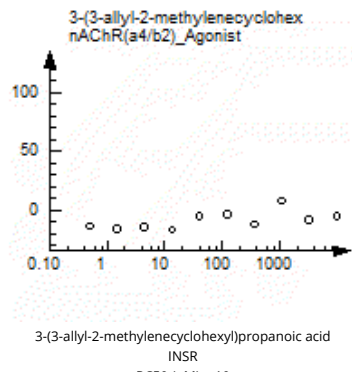
$R C 50(u M)>10$

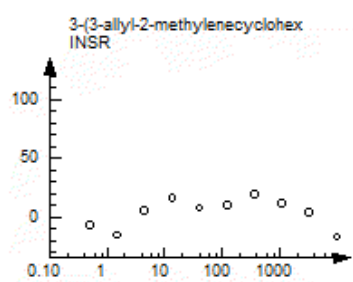

3-(3-allyl-2-methylenecyclohexyl)propanoic acid GABAA Agonist
$\mathrm{RC} 50(\mathrm{UM})>10$

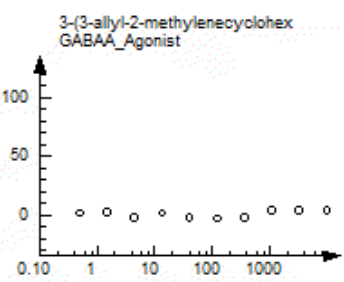

3-(3-ally1-2-methylenecyclohexyl)propanoic acid KVLQT1/minK Antagonist

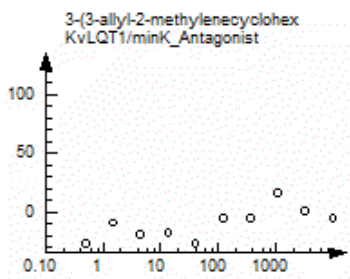

3-(3-allyl-2-methylenecyclohexyl)propanoic acid RC50 (UM) $>10$
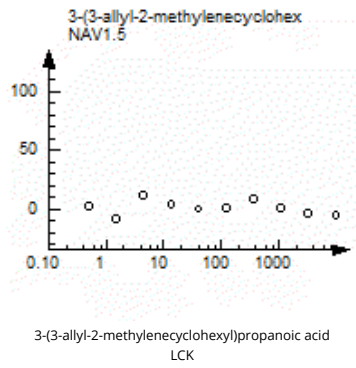
$\mathrm{RC}(\mathrm{UM})>10$

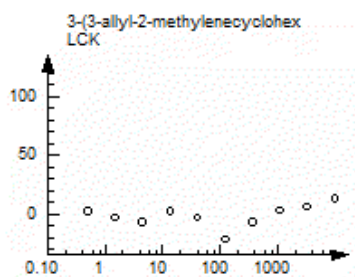

3-(3-allyl-2-methylenecyclohexyl)propanoic acid
hERG RCSO (UM) $>10$

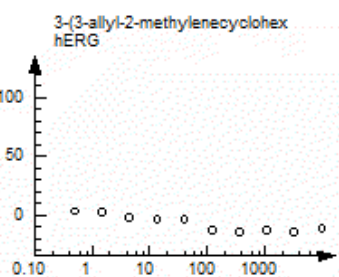

3-(3-allyl-2-methylenecyclohexyl)propanoic acid KvLQT1/mink Agonist
RC50 (uM) $>10$

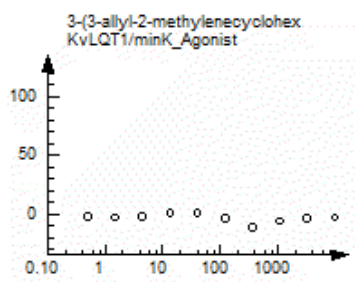

3-(3-allyl-2-methylenecyclohexyl)propanoic acid

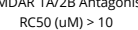

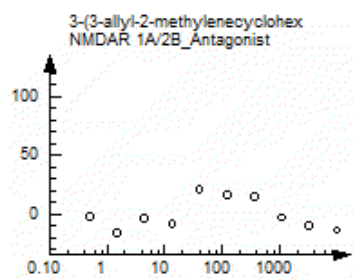

3-(3-allyl-2-methylenecyclohexyl)propanoic acid
ROCK1 $\mathrm{RC} 50(\mathrm{UM})>10$

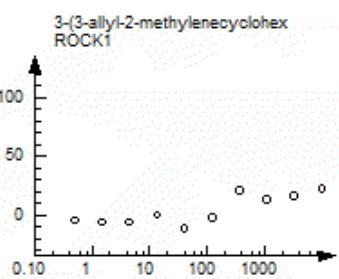


Figure Sup. 1 (cont.)
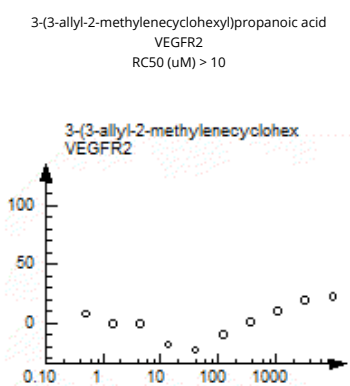

3-(3-allyl-2-methylenecyclohexyl)propanoic acid GR Antagonist
RC50 (UM) $>10$
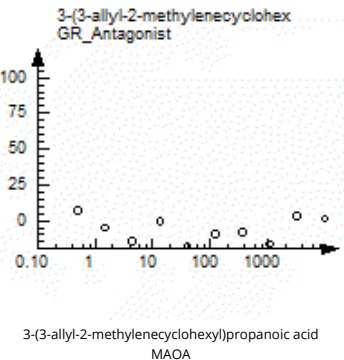
$\mathrm{RC} 50(\mathrm{UM})>10$
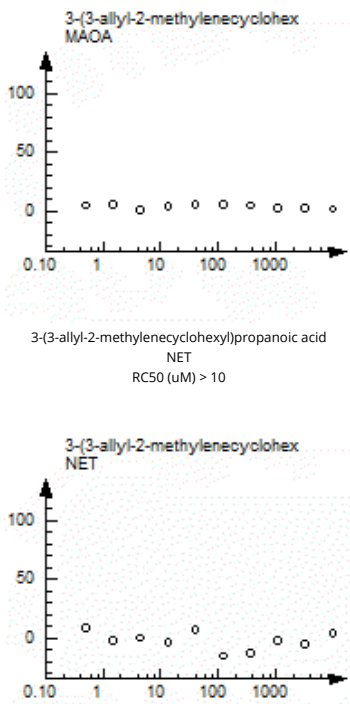
3-(3-allyl-2-methylenecyclohexyl)propanoic acid
AR Agonist

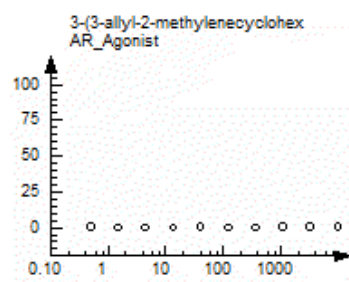

3-(3-allyl-2-methylenecyclohexyl)propanoic acid AChE
$\mathrm{RC50}(\mathrm{UM})>10$

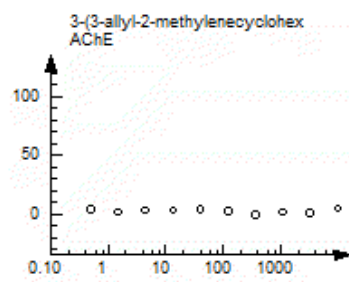

3-(3-allyl-2-methylenecyclohexyl)propanoic acid $\mathrm{RC} 5$ (UM) $>10$

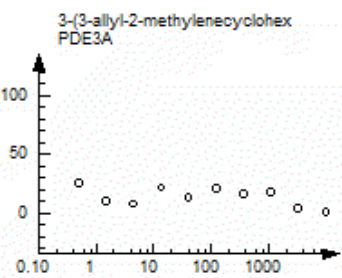

3-(3-ally-2-methylenecyclohexyl)propanoic acid
SERT $\mathrm{RC} 50(\mathrm{UM})>10$

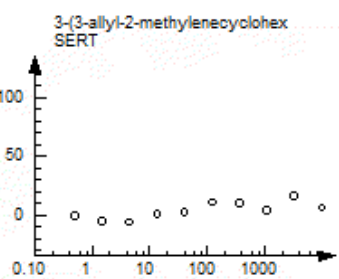

3-(3-allyl-2-methylenecyclohexyl)propanoic acid
AR Antagonist AR Antagonist
$\mathrm{RC} 50(\mathrm{UM})>10$

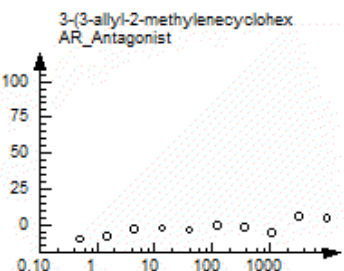

3-(3-allyl-2-methylenecyclohexyl)propanoic acid $\operatorname{COX} 1$
$\mathrm{RC} 50(\mathrm{MM})>10$

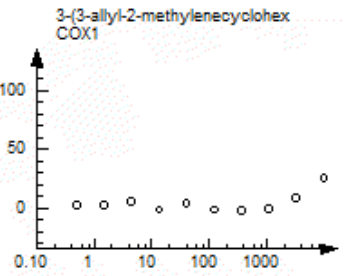

3-(3-allyl-2-methylenecyclohexyl)propanoic acid PDE4D2
$\operatorname{RC50}($ (UM) $>10$

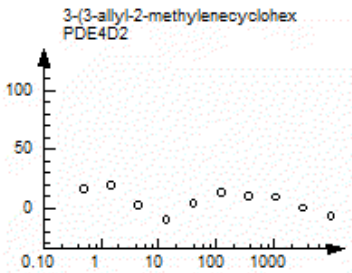

3-(3-allyl-2-methylenecyclohexyl)propanoic acid GR Agonist
$R C 50(u M)>10$

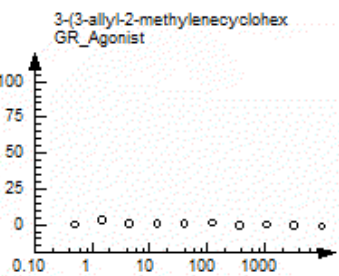

3-(3-allyl-2-methylenecyclohexyl)propanoic acid $\mathrm{COX} 2$
$\mathrm{RC} 50(\mathrm{UM})>10$

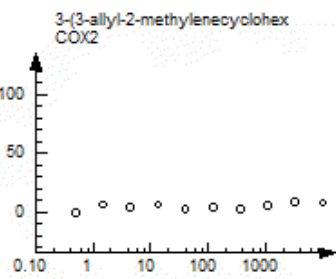

3-(3-allyl-2-methylenecyclohexyl)propanoic acid
DAT $\mathrm{RC} 50(\mathrm{UM})>10$

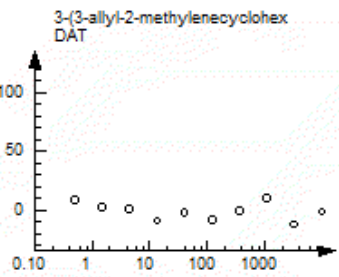




\section{MCA-13 safety by in vitro evaluation using PhosphoSens ${ }^{\circledR C C S o x b a s e d ~ k i n a s e ~}$ and phosphatase assays}

\section{Method: Kinase Activity Measurements}

Kinase activity was measured continuously using the PhosphoSens ${ }^{\circledR}$ technology (AssayQuant Technologies Inc., Marlborough, MA) according to the manufacturers' recommendations (http://www.assayquant.com/) . This one-step homogeneous assay uses chelation-enhanced fluorescence via optimized substrate sensors unique for each kinase, containing the unnatural fluorogenic amino-acid Sox.

\section{Recombinant Kinases.}

The following kinases which, upon stimulation or inhibition with small molecule modulators, induced neurite outgrowth, were obtained from different companies and were used in this study at the following concentrations: $2.0 \mathrm{nM}$ TRKA, amino acids 442-796, BPS Bio (cat/lot \# 40280/151113G)/ AQT0101 1.0 nM TRKB, amino acids 456-822, BPS Bio (cat. \& lot \#: 40281, 130826)/ AQT0101 1.0 nM TRKC, amino acids 456-825, BPS Bio (cat. \& lot \#: 40282/140205G2)/ AQT0101 1.0 nM CDK5/p25, amino acids 1-292/99-307, Carna (cat. \& lot \#: 04-106/09CBS0884K)/ AQT0255 5.0 nM ASK1, amino acids 1-1374, Invitrogen (cat. \& lot \#: PV3809/1965588C)/ AQT0557 20 nM BTN-DLK, amino acids 1-520, Carna (cat. \& lot \#: 09411-20N, 11CBS-0859B)/ AQT0492 5.0 nM JNK3, amino acids 1-426, Carna (cat. \& lot \#: 04150, 09CBS-0155G)/ AQT0371 2.5 nM ERK2, amino acids 1-360, Carna (cat. \& lot \#: 04143/12CBS-0211N)/ AQT0490 $5.0 \mathrm{nM} \mathrm{p38 \alpha ,} \mathrm{amino} \mathrm{acids} \mathrm{9-352,} \mathrm{Carna} \mathrm{(cat.} \mathrm{\&} \mathrm{lot} \mathrm{\# :} \mathrm{04-152,}$ 07CBS-0319H)/ AQT0376 5.0 nM nM PDGFR $\beta$, amino acids 557-1106, Carna (cat. \& lot \#: 08158, 08CBS-0282H)/ AQT0101 5.0 nM IKK $\beta$, amino acids 1-756, Invitrogen (cat/lot \# PV3836 / 1311416G)/ AQT0215 $2.0 \mathrm{nM}$ AKT1, amino acids 104-480, Carna (cat. \& lot \#: 01-101, 13CBS0155F)/ AQT0569 0.50 nM GSK3 $\beta$, amino acids 1-420, Carna (cat. \& lot \#: 04-141/13CBS0346M)/ AQT0320 1.0 nM PKCE, amino acids 1-737, Carna (cat. \& lot \#: 01-136/09CBS-0150E)/ AQT0462 1.0 nM PKCl, amino acids 1-587, Carna (cat. \& lot \#: 01-139, 09CBS-162F)/ AQT0462 $1.0 \mathrm{nM}$ PKAC $\alpha$, amino acids 1-351, Carna (cat. \& lot \#: 01-127/10CBS-0315N)/ AQT0458 2.5 nM KDR, amino acids 790-1356, Carna (cat. \& lot \#: 08-191, 13CBS-0442G)/ AQT00001 $1.0 \mathrm{nM}$ 
nM CK1 $\delta$, amino acids 1-294, Carna (cat. \& lot \#: 03-103, 09CBS-1197J)/ ST24; 5.0 nM EGFR, amino acids 668-1210, BPS Bio (cat. \& lot \#: 40187, 150312-G2)/ AQT0001 2.0 nM IGF1R, amino acids 959-1367, Carna (cat. \& lot \#: 08-141/07CBS-0753B)/ AQT0101 1.0 nM CAMK2 $\alpha$, amino acids 1-478, Carna (cat. \& lot \#: 02-109/11CBS-0288K)/ AQT0425 1.0 nM FGFR2, amino acids 399-821, Carna (cat. \& lot \#: 08-134/13CBS-0735G)/ AQT0001 1.0 nM FGFR1, amino acids 396-820, Carna (cat. \& lot \#: 08-133, 12CBS-0123L)/ AQT0001

\section{Reaction Set Up}

$25 \mu \mathrm{L}$ final reaction volume including : $2.5 \mu$ Sox-based substrate $(10 \mathrm{x}), 17.5 \mu 1$ reaction mix with ATP, $5.0 \mu \mathrm{L}$ Kinase [5x in EDB (Enzyme Dilution Buffer : 20 mM TRIS, pH 8.0, 0.01\% Brij-35, $0.1 \mathrm{mM}$ EGTA, 5\% Glycerol, $1 \mathrm{mM}$ DTT, $1 \mathrm{mg} / \mathrm{mL}$ Bovine Serum Albumin]. 5-45 min preincubation (all except EDB/kinase) at $30{ }^{\circ} \mathrm{C}$ of substrate in reaction mix and ATP ensuring that the progress kinetic curve for the background fluorescence was flat. Reaction was run at $30{ }^{\circ} \mathrm{C}$ for either 120 or 180 minutes.

\section{Final Reaction Conditions.}

Protein kinase activity was determined in 54 mM HEPES, pH $7.51 \mathrm{mM}$ ATP $1.2 \mathrm{mM}$ DTT (none in ASK1) $0.012 \%$ Brij-35 1\% glycerol $0.2 \mathrm{mg} / \mathrm{ml}$ BSA $0.52 \mathrm{mM}$ EGTA (none in assays of CAMK2 $\alpha, \mathrm{PKC} \varepsilon, \mathrm{PKCl}$, Calcineurin A/B) $140 \mu \mathrm{M}$ phosphatidylserine/3.8 $\mu \mathrm{M}$ diacylglycerol (PKC $\varepsilon, \mathrm{PKCl}$ only) $5 \mathrm{ng} / \mu \mathrm{l}$ calmodulin/ $0.4 \mathrm{mM} \mathrm{CaCl}_{2}$ (CAMK2 $\alpha$, Calcineurin A/B only) $10 \mathrm{mM}$ $\mathrm{MgCl}_{2} 15 \mu \mathrm{M}$ Substrate (ST24, AQT0001, 101, 215, 255, 320, 371, 376, 425, 462, 458, 490, 492, 557, 569) 0.50-20 nM Kinase (TRKA, TRKB, TRKC, CDK5/p25, JNK3, ERK2, p38 $\alpha$, PDGFR $\beta$, IKK $\beta$, AKT1, GSK3 $\beta$, PKC $\varepsilon$, PKC1, PKAC $\alpha$, KDR, CK1 $\delta$, EGFR, ASK1, IGF1R, CAMK2 $\alpha$, DLK, FGFR2, FGFR1) in the presence or absence of $5 \mu \mathrm{M}$ HU-MCA-13 test compound. All components except enzyme were equilibrated to $30{ }^{\circ} \mathrm{C}$ prior to setting up reactions run in Corning, 384-well, white flat round bottom polystyrene NBS microplates (Cat. \#3824) after sealing using optically-clear adhesive film [TopSealA-Plus plate seal, PerkinElmer (Cat. \#6050185)], applied with a roller to eliminate evaporation and resulting drift. Europium was added to a final concentration of $5 \mathrm{mM}$ and the reaction was stopped by $2.5 \mu \mathrm{M} \mathrm{MCl}$ for 15 minutes, followed by neutralization with $2.5 \mu \mathrm{l} 1 \mathrm{M} \mathrm{NaOH}$ for additional 15 minutes. Thereafter, the fluorescence intensity was kinetically read every 2 minutes from the top for up to 120 minutes at $30{ }^{\circ} \mathrm{C}$, with excitation and emission wavelengths of $360 \mathrm{~nm}$ and $485 \mathrm{~nm}$, respectively, using a Synergy Neo2 
multi-mode plate reader (Biotek Instruments, Winooski, VT). Fluorescence, determined with identical reactions but lacking enzyme was subtracted from the total fluorescence signal for each time point, with both determined in duplicate, to obtain corrected relative fluorescence units (RFU). Corrected RFU values then were plotted versus time and the reaction velocity for the first $\sim 40$ minutes (initial reaction rates) were determined from the slope using GraphPad Prism (La Jolla, CA) with units of RFU/min.

\section{Results}

Figure Sup. 2 - Progress curves for kinases that stimulate neurite outgrowth

TRKA-C +/- 5 $\mu$ M MCA-13 Activator
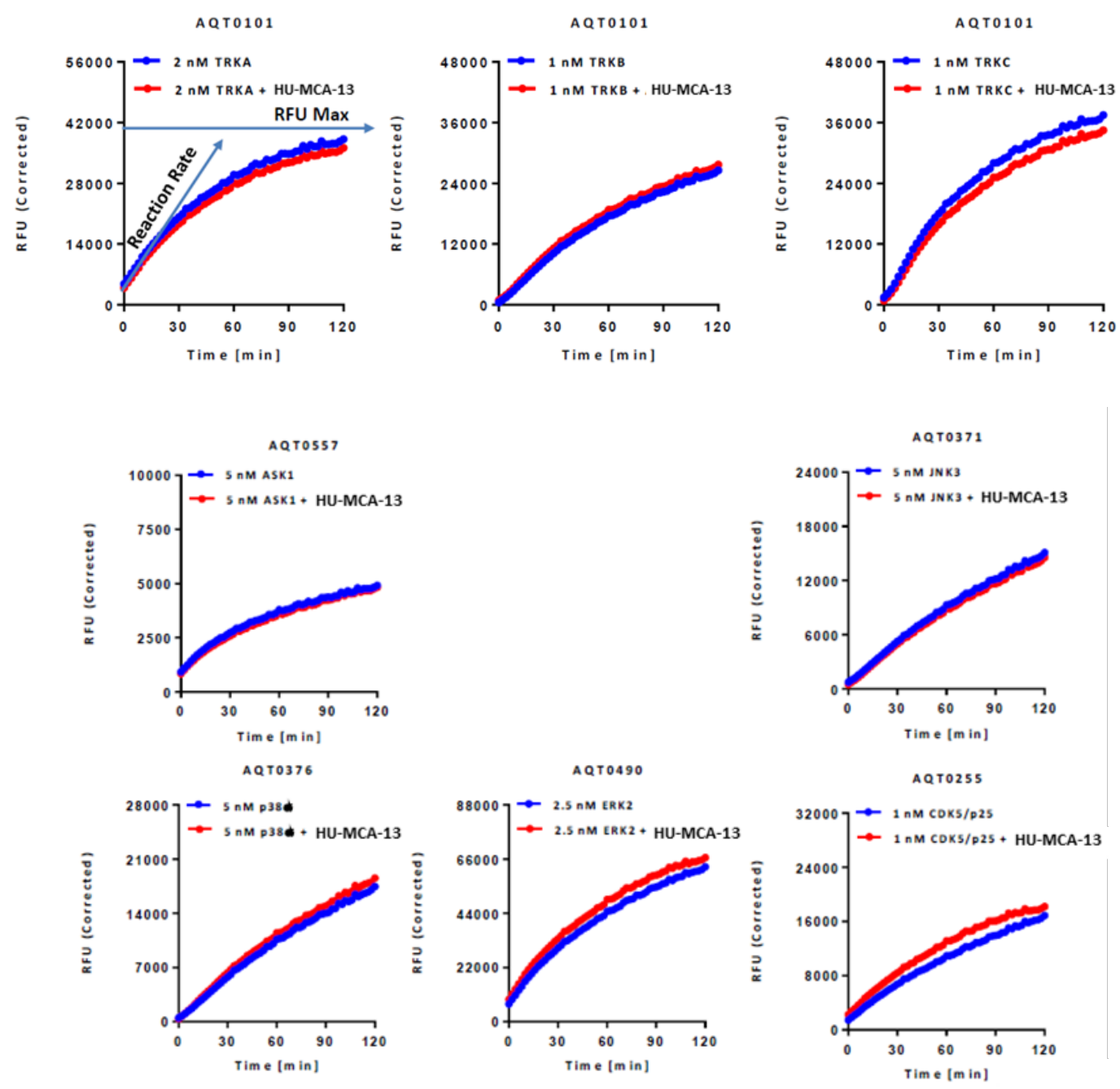
Figure Sup. 2 (cont.)
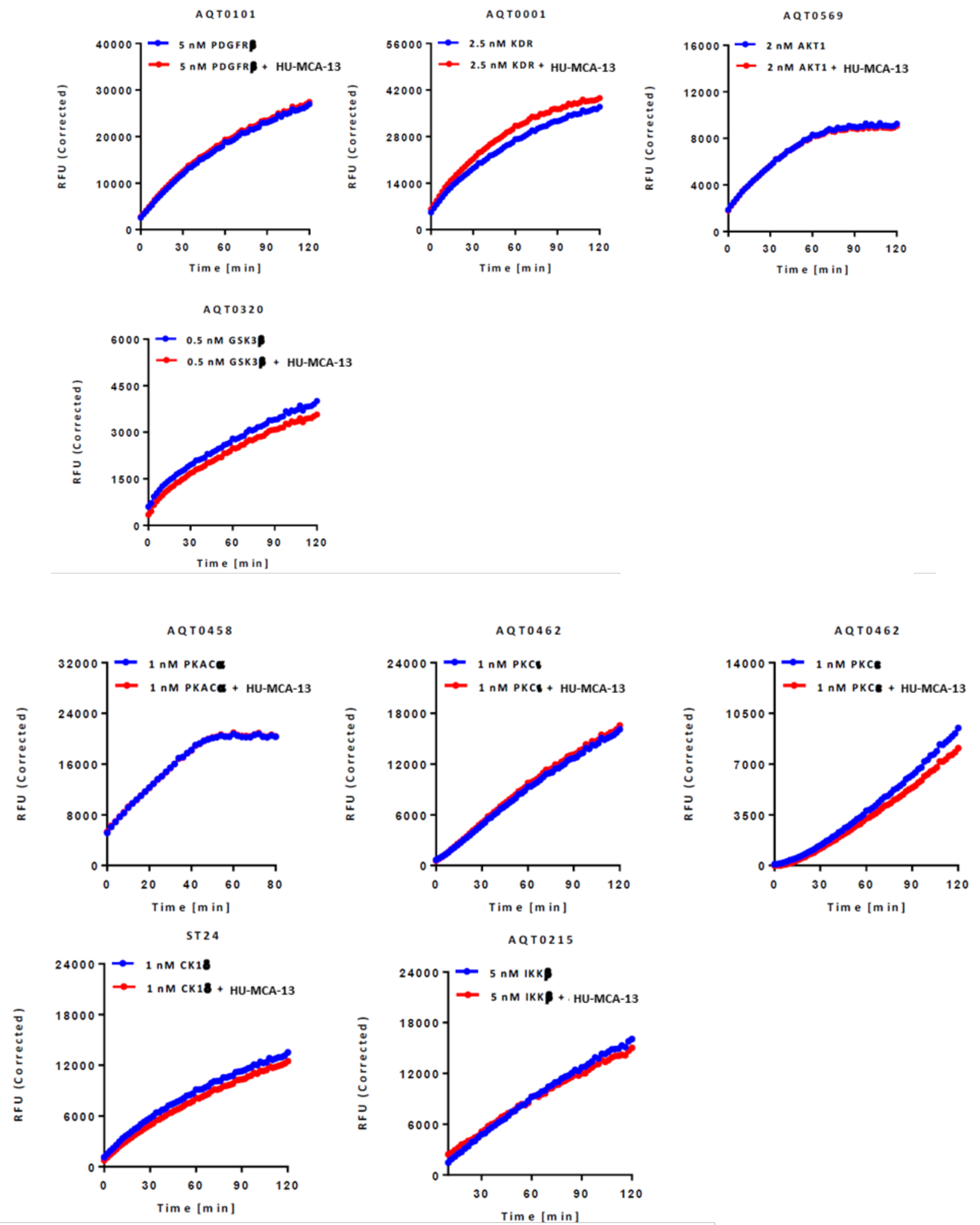
Figure Sup. 2 (cont.)

\section{Progress curves for kinases that inhibit neurite outgrowth}
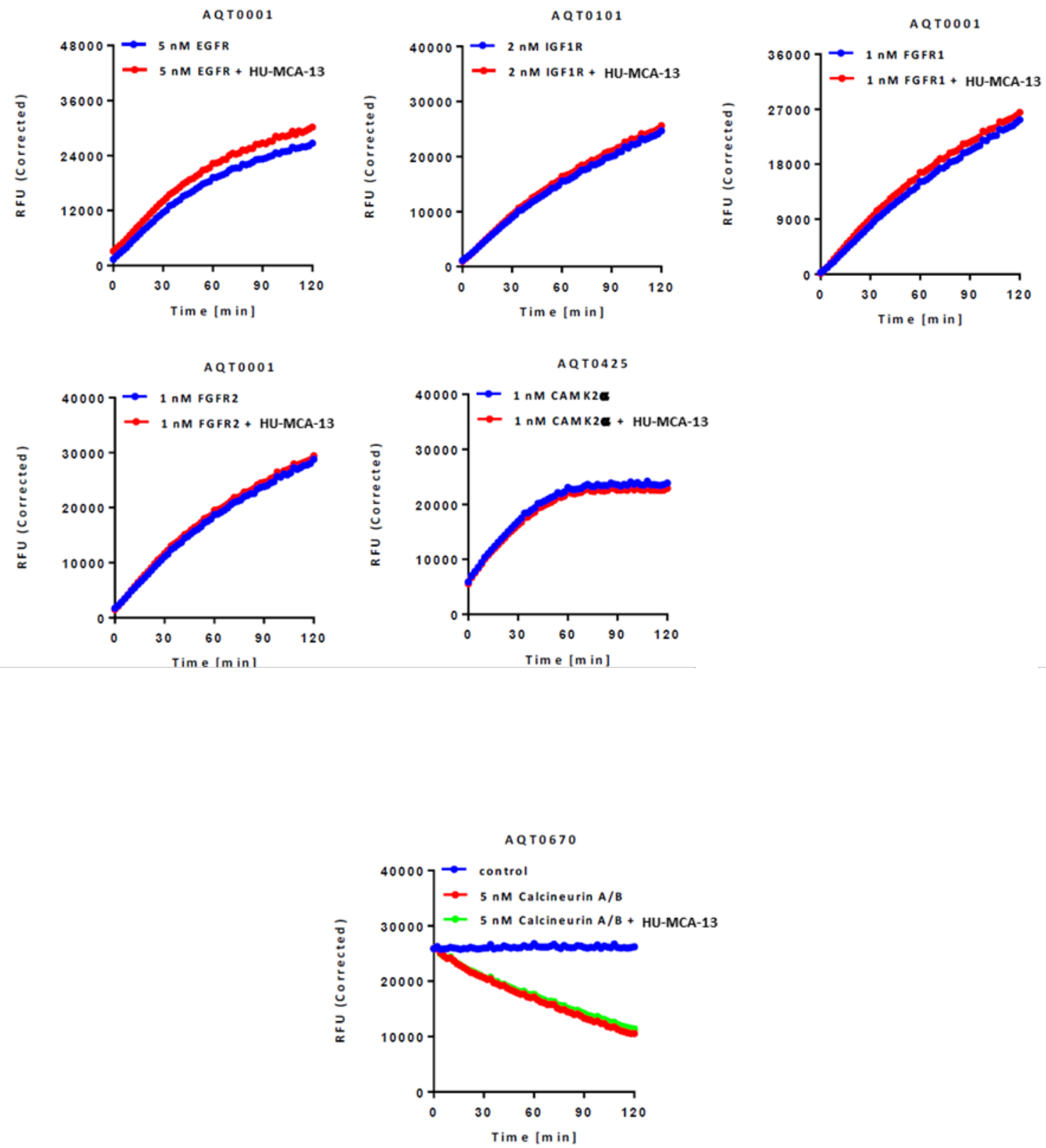